\title{
Wirtinger inequality using Bessel functions
}

\section{Tatjana Z. Mirković ${ }^{*}$}

"Correspondence:

tatjana.mirkovic@visokaskola.edu.rs

${ }^{1}$ College of Applied Professional

Studies, Vranje, Serbia

\section{Abstract \\ This paper presents of some new Wirtinger-type integral inequalities by using Bessel functions. We establish one weighted Wirtinger inequality.}

MSC: 26D20; 33C10

Keywords: Wirtinger's inequality; Bessel functions

\section{Introduction}

The Wirtinger inequality plays a very important role in the theory of approximation, the theory of Sobolev's spaces, the theory of function of several variables and functional analysis. In 1916. Wirtinger established an integral inequality.

Theorem 1.1 (Wirtinger inequality) Let $f: \mathbb{R} \rightarrow \mathbb{R}$ be a continuous periodic function with period $2 \pi$ and let $f^{\prime} \in L^{2}$. Then, if $\int_{0}^{2 \pi} f(x) d x=0$ the following inequality holds:

$$
\int_{0}^{2 \pi} f^{2}(x) d x \leq \int_{0}^{2 \pi} f^{\prime 2}(x) d x
$$

with equality if and only if $f(x)=a \cos x+b \sin x$, where $a$ and $b$ are constants.

Theorem 1.2 Let $f(x)$ be a smooth function with period $2 \pi$. Then, for all real $t$,

$$
\int_{0}^{2 \pi}[f(x)-f(x+t)]^{2} d x \leq 4 \sin ^{2} \frac{t}{2} \int_{0}^{2 \pi} f^{\prime 2}(x) d x .
$$

Equality is attained if and only if $f(x)=a \cos x+b \sin x+c$, where $a, b, c$ are real constants (for $t=0$ equality holds always).

In [1], Beesack obtained the following generalization of the Wirtinger inequality: If $k>1$, $f(x) \in C^{1}([0, \pi]), f(0)=0$, then

$$
\int_{0}^{\pi}\left(f^{\prime}(x)\right)^{2 k} d x \geq \frac{2 k-1}{\left(k \sin \frac{\pi}{2 k}\right)^{2 k}} \int_{0}^{\pi} f^{2 k}(x) d x, \quad k \geq 1
$$

In [2], Hall proved the following theorem:

(c) The Author(s) 2018. This article is distributed under the terms of the Creative Commons Attribution 4.0 International License (http://creativecommons.org/licenses/by/4.0/), which permits unrestricted use, distribution, and reproduction in any medium, provided you give appropriate credit to the original author(s) and the source, provide a link to the Creative Commons license, and indicate if changes were made. 
Theorem 1.3 Suppose that $k \in N, f(x) \in C^{2}[0, \pi]$ and $f(0)=f(\pi)=0$. Let $H(u)$ be an even function, increasing and strictly convex on $R^{+}$, and such that $H(0)=H^{\prime}(0)=0$; moreover, $u H^{\prime \prime}(u) \rightarrow 0$ as $u \rightarrow 0$. Then we have

$$
\int_{0}^{\pi} H\left(f^{\prime}(x) / f(x)\right) f^{2 k}(x) \geq(2 k-1) \lambda \int_{0}^{\pi} f^{2 k}(x) d x, \quad \lambda=\lambda(k, H),
$$

where $\lambda=\lambda(k, H)$ is determined by the equation

$$
\int_{0}^{\infty} \frac{G^{\prime}(u)}{G(u)+(2 k-1) \lambda} \frac{d u}{u}=k \pi, \quad G(u):=u H^{\prime}(u)-H(u) .
$$

For each non-negative constant $p$, the associated Bessel equation is

$$
x^{2} \frac{d^{2} y}{d x^{2}}+x \frac{d y}{d x}+\left(x^{2}-p^{2}\right) y=0
$$

Since Bessel's differential equation is a second-order equation, there must be two linearly independent solutions, which are called Bessel functions. These functions play important roles in many areas of applied mathematics (see [3, 4]). Typically the general solution is given as

$$
y=a_{1} J_{p}(x)+a_{2} Y_{p}(x)
$$

where $a_{1}$ and $a_{2}$ are arbitrary constants.

Special functions $J_{p}(x)$ are Bessel functions of the first kind, which are finite at $x=0$ for all real values of $p$, and $Y_{p}(x)$ are Bessel functions of the second kind, which are singular at $x=0$.

The Bessel function of the first kind of order $p$ can be determined using an infinite power series expansion as follows: $J_{p}(x)=\sum_{k=0}^{+\infty} \frac{(-1)^{k}}{k ! \Gamma(k+p+1)}\left(\frac{x}{2}\right)^{2 k+p}$. Since $\Gamma(k+1)=k !$, it follows that

$$
J_{p}(x)=\sum_{k=0}^{+\infty} \frac{(-1)^{k}}{k !(k+p) !}\left(\frac{x}{2}\right)^{2 k+p} .
$$

For integer order $p$, functions $J_{p}$ and $J_{-p}$ are not linearly independent, $J_{-p}=(-1)^{p} J_{p}$. In contrast, for non-integer orders, $J_{p}$ and $J_{-p}$ are linearly independent.

The most important Bessel functions are $J_{0}(x)$ and $J_{1}(x)$. For $p=-\frac{1}{2}$ and $p=\frac{1}{2}$, this functions expansion as follows:

$$
\begin{aligned}
& J_{-1 / 2}(x)=\sqrt{\frac{2}{\pi x}} \cos x, \\
& J_{1 / 2}(x)=\sqrt{\frac{2}{\pi x}} \sin x .
\end{aligned}
$$

\section{Main results}

Theorem 2.1 Let $f^{\prime} \in L^{2 k}$ on $[0, \pi]$, with $f(0)=f(\pi)=0$. Then the following inequality holds:

$$
\int_{0}^{\pi} f^{2 k}(x) d x \leq \frac{1}{2 k-1}\left(\frac{\pi}{2}\right)^{2 k}\left(\int_{0}^{\frac{\pi}{2}} J_{0}\left(\frac{\pi}{2 k} \cos t\right) \cos t d t\right)^{2 k} \int_{0}^{\pi} f^{\prime 2 k}(x) d x .
$$


Proof Since $J_{n}(z)=\left(\frac{z}{2}\right)^{n} \sum_{r=0}^{+\infty}(-1)^{r} \frac{\left(\frac{z}{2}\right)^{2 r}}{r !(n+r) !}$, it follows that $J_{0}\left(\frac{\pi}{2 k} \cos t\right)=\sum_{r=0}^{+\infty}(-1)^{r} \frac{\left(\frac{\pi}{2 k} \frac{\cos t}{2}\right)^{2 r}}{(r !)^{2}}$.

$$
\begin{aligned}
\int_{0}^{\frac{\pi}{2}} J_{0}\left(\frac{\pi}{2 k} \cos t\right) \cos t d t & =\int_{0}^{\frac{\pi}{2}} \sum_{r=0}^{+\infty}(-1)^{r} \frac{\left(\frac{\pi}{2 k} \frac{\cos t}{2}\right)^{2 r}}{(r !)^{2}} \cos t d t \\
& =\sum_{r=0}^{+\infty}(-1)^{r} \frac{\pi^{2 r}}{(2 k)^{2 r} 2^{2 r}(r !)^{2}} \int_{0}^{\frac{\pi}{2}} \cos ^{2 r+1} t d t
\end{aligned}
$$

Using the integration by parts formula on the integral $I_{2 r+1}=\int_{0}^{\frac{\pi}{2}} \cos ^{2 r+1} t d t$ and the fact that $\int_{0}^{\pi / 2} \cos t d t=1$, we obtained the recurrence relation $I_{2 r+1}=\frac{2 r}{2 r+1} I_{2 r-1}$, which implies $I_{2 r+1}=\frac{(2 r) ! !}{(2 r+1) ! !}=\frac{((2 r) ! !)^{2}}{(2 r+1) !}=\frac{\left(2^{r} r !\right)^{2}}{(2 r+1) !}$.

The above equality becomes

$$
\begin{aligned}
\int_{0}^{\frac{\pi}{2}} J_{0}\left(\frac{\pi}{2 k} \cos t\right) \cos t d t & =\sum_{r=0}^{+\infty}(-1)^{r} \frac{\pi^{2 r}}{(2 k)^{2 r} 2^{2 r}(r !)^{2}} \frac{\left(2^{r} r !\right)^{2}}{(2 r+1) !} \\
& =\frac{2 k}{\pi} \sum_{r=0}^{+\infty}(-1)^{r} \frac{\left(\frac{\pi}{2 k}\right)^{2 r+1}}{(2 r+1) !}=\frac{2 k}{\pi} \sin \frac{\pi}{2 k}
\end{aligned}
$$

which implies

$$
\sin ^{2 k} \frac{\pi}{2 k}=\left(\frac{\pi}{2 k}\right)^{2 k}\left(\int_{0}^{\frac{\pi}{2}} J_{0}\left(\frac{\pi}{2 k} \cos t\right) \cos t d t\right)^{2 k} .
$$

By (2) it follows that

$$
\begin{aligned}
& \int_{0}^{\pi} f^{2 k}(x) d x \\
& \quad \leq \frac{1}{2 k-1} k^{2 k} \frac{\pi^{2 k}}{4^{k} k^{2 k}}\left(\int_{0}^{\frac{\pi}{2}} J_{0}\left(\frac{\pi}{2 k} \cos t\right) \cos t d t\right)^{2 k} \int_{0}^{\pi} f^{\prime 2 k}(x) d x, \\
& \int_{0}^{\pi} f^{2 k}(x) d x \\
& \quad \leq \frac{1}{2 k-1}\left(\frac{\pi}{2}\right)^{2 k}\left(\int_{0}^{\frac{\pi}{2}} J_{0}\left(\frac{\pi}{2 k} \cos t\right) \cos t d t\right)^{2 k} \int_{0}^{\pi} f^{\prime 2 k}(x) d x .
\end{aligned}
$$

Theorem 2.2 If $f^{\prime} \in L^{2 k}$ is absolutely continuous on $[0, \pi]$, with $f(0)=f(\pi)=0$ then

$$
\int_{0}^{\pi} f^{2 k}(x) d x \leq \frac{\pi^{2 k}}{2 k+1} C(k) \int_{0}^{\pi} f^{\prime 2}(x) f^{2(k-1)}(x) d x
$$

where $C(k):=\frac{\int_{0}^{\frac{\pi}{2}} x^{k} J_{1 / 2}^{2 k}(x) d x}{\int_{0}^{\frac{\pi}{2}} x^{k+1} J_{1 / 2}^{2 k+1}(x) d x}$.

Proof Starting with the right side of (10), we obtain

$$
\begin{aligned}
& \frac{\pi^{2 k}}{2 k+1} C(k) \int_{0}^{\pi} f^{\prime 2}(x) f^{2(k-1)}(x) d x \\
& \quad=\frac{\pi^{2 k}}{2 k+1} \frac{\int_{0}^{\frac{\pi}{2}} x^{k} J_{1 / 2}^{2 k}(x) d x}{\int_{0}^{\frac{\pi}{2}} x^{k+1} J_{1 / 2}^{2 k+1}(x) d x} \int_{0}^{\pi} f^{\prime 2}(x) f^{2(k-1)}(x) d x
\end{aligned}
$$




$$
\begin{aligned}
& =\frac{\pi^{2 k} \int_{0}^{\frac{\pi}{2}} x^{k}\left(\sqrt{\frac{2}{\pi x}}\right)^{2 k} \sin ^{2 k} x d x}{(2 k+1) \int_{0}^{\frac{\pi}{2}} x^{k+1}\left(\sqrt{\frac{2}{\pi x}}\right)^{2 k+1} \sin ^{2 k+1} x d x} \int_{0}^{\pi} f^{\prime 2}(x) f^{2(k-1)}(x) d x \\
& =\frac{\pi^{2 k}}{2 k+1} \sqrt{\frac{\pi}{2}} \frac{\int_{0}^{\frac{\pi}{2}} \sin ^{2 k} x d x}{\int_{0}^{\frac{\pi}{2}} \sin ^{2 k+1} x d x} \int_{0}^{\pi} f^{\prime 2}(x) f^{2(k-1)}(x) d x .
\end{aligned}
$$

Since $\int_{0}^{\frac{\pi}{2}} \sin ^{p} x \cos ^{q} x d x=\frac{\Gamma\left(\frac{p+1}{2}\right) \Gamma\left(\frac{q+1}{2}\right)}{2 \Gamma\left(\frac{p+q}{2}+1\right)}$, for $p=2 k$ and $q=0$, we get $\int_{0}^{\frac{\pi}{2}} \sin ^{2 k} x d x=$ $\frac{\Gamma\left(\frac{1}{2}\right) \Gamma\left(\frac{2 k+1}{2}\right)}{2 \Gamma(k+1)}$; For $p=2 k+1$ and $q=0$, we get $\int_{0}^{\frac{\pi}{2}} \sin ^{2 k+1} x d x=\frac{\Gamma\left(\frac{1}{2}\right) \Gamma(k+1)}{2 \Gamma\left(\frac{2 k+3}{2}\right)}$.

By integrating by parts, we obtain $\Gamma\left(\frac{2 k+1}{2}\right)=\frac{(2 k) !}{2^{k} k !} \sqrt{\pi}, \Gamma\left(\frac{2 k+3}{2}\right)=\frac{(2 k+1) !}{2^{2 k+1} k !} \sqrt{\pi}$, and since $\Gamma\left(\frac{1}{2}\right)=\sqrt{\pi}, \Gamma(k+1)=k !$, it follows

$$
\begin{aligned}
& \frac{\pi^{2 k}}{2 k+1} \sqrt{\frac{\pi}{2}} \frac{\frac{\pi}{2} \frac{(2 k) !}{\frac{(k !))^{2}}{(2 k+1) !}}}{{ }^{2 k}(k)} f_{0}^{\prime 2}(x) f^{2(k-1)}(x) d x \\
& =\frac{1}{2 k+1}\left(\sqrt{\frac{\pi}{2}}\right)^{2 k+\frac{3}{2}} \frac{((2 k) !)^{2}(2 k+1)}{\left(2^{k} k !\right)^{2}(k !)^{2}} \int_{0}^{\pi} f^{\prime 2}(x) f^{2(k-1)}(x) d x \\
& =\frac{1}{2 k+1}\left(\sqrt{\frac{\pi}{2}}\right)^{2 k+\frac{3}{2}}\left(\frac{(2 k-1) !}{k(k !)}\right)^{2} k^{2} \int_{0}^{\pi} f^{\prime 2}(x) f^{2(k-1)}(x) d x
\end{aligned}
$$

If in (4) we put $H(u)=u^{2}, G(u)=u^{2}$, then (6) gives $\lambda=\frac{1}{k^{2}(2 k-1)}$, so (3) becomes

$$
\int_{0}^{\pi} f^{\prime 2}(x) f^{2(k-1)}(x) d x \geq \frac{1}{k^{2}} \int_{0}^{\pi} f^{2 k}(x) d x
$$

which implies

$$
\frac{\pi^{2 k}}{2 k+1} C(k) \int_{0}^{\pi} f^{\prime 2}(x) f^{2(k-1)}(x) d x \geq\left(\sqrt{\frac{\pi}{2}}\right)^{2 k+\frac{3}{2}}\left(\frac{(2 k-1) !}{k(k !)}\right)^{2} \int_{0}^{\pi} f^{2 k}(x) d x .
$$

Since $\left(\sqrt{\frac{\pi}{2}}\right)^{2 k+\frac{3}{2}}>1$ and $\left(\frac{(2 k-1) !}{k(k !)}\right)^{2}>1$, inequality $(10)$ is established.

Theorem 2.3 Let $f(x)$ be a smooth function with period $2 \pi$. Then, for all real $t$,

$$
\int_{0}^{2 \pi}[f(x)-f(x+t)]^{2} d x \leq t \pi J_{1 / 2}^{2}\left(\frac{t}{2}\right) \int_{0}^{2 \pi} f^{\prime 2}(x) d x .
$$

Equality is attained if and only iff $(x)=A \cos x+B \sin x+C$, where $A, B, C$ are real constants (for $t=0$ equality holds always).

Proof From the equation $J_{n}^{2}(t)=\frac{2}{\pi} \int_{0}^{\frac{\pi}{2}} J_{2 n}(2 t \cos x) d x$, for $n=\frac{1}{2}$, the right side of (11) becomes

$$
\begin{aligned}
& 2 t \int_{0}^{\frac{\pi}{2}} J_{1}(t \cos x) d x \int_{0}^{2 \pi} f^{\prime 2}(x) d x \\
& \quad=2 t \int_{0}^{\frac{\pi}{2}} \sum_{n=0}^{+\infty} \frac{(-1)^{n}}{n !(n+1) !}\left(\frac{t \cos x}{2}\right)^{2 n+1} d x \int_{0}^{2 \pi} f^{\prime 2}(x) d x
\end{aligned}
$$




$$
\begin{aligned}
& =2 t \sum_{n=0}^{+\infty} \frac{(-1)^{n}}{n !(n+1) !}\left(\frac{t}{2}\right)^{2 n+1} \int_{0}^{\frac{\pi}{2}} \cos ^{2 n+1} x d x \int_{0}^{2 \pi} f^{\prime 2}(x) d x \\
& =2 t \sum_{n=0}^{+\infty} \frac{(-1)^{n}}{n !(n+1) !} \frac{t^{2 n+1}}{2 \cdot 2^{2 n}} \frac{2^{2 n} n ! n !}{(2 n+1) !} \int_{0}^{2 \pi} f^{\prime 2}(x) d x \\
& =t \sum_{n=0}^{+\infty} \frac{(-1)^{n} n ! t^{2 n+1}}{(n+1) n !(2 n+1) !} \int_{0}^{2 \pi} f^{\prime 2}(x) d x \\
& =t \sum_{n=0}^{+\infty} \frac{(-1)^{n} t^{2 n+1}}{(n+1)(2 n+1) !} \int_{0}^{2 \pi} f^{\prime 2}(x) d x \\
& =t\left[\frac{t}{1 !}-\frac{t^{3}}{2 \cdot 3 !}+\frac{t^{5}}{3 \cdot 5 !}-\frac{t^{7}}{4 \cdot 7 !}+\cdots\right] \int_{0}^{2 \pi} f^{\prime 2}(x) d x \\
& =t\left[\frac{2 t^{2}}{2 ! t}-\frac{4 t^{4}}{2 \cdot 4 ! t}+\frac{6 t^{6}}{3 \cdot 6 ! t}-\frac{8 t^{8}}{4 \cdot 8 ! t}+\frac{10 t^{10}}{5 \cdot 10 ! t}-\cdots\right] \int_{0}^{2 \pi} f^{\prime 2}(x) d x \\
& =2 t\left[\frac{t^{2}}{2 ! t}-\frac{t^{4}}{4 ! t}+\frac{t^{6}}{6 ! t}-\frac{t^{8}}{8 ! t}+\frac{t^{10}}{10 ! t}-\cdots\right] \int_{0}^{2 \pi} f^{\prime 2}(x) d x \\
& =2 t \sum_{n=1}^{+\infty}(-1)^{n+1} \frac{t^{2 n}}{t(2 n) !} \int_{0}^{2 \pi} f^{\prime 2}(x) d x \\
& =t\left(\sum_{n=0}^{+\infty}(-1)^{n} \frac{t^{2 n}}{(2 n) !}\right) \int_{0}^{2 \pi} f^{\prime 2}(x) d x \\
& =
\end{aligned}
$$

Equation (1) implies the desired inequality (11).

\section{Acknowledgements}

The author would like to thank the anonymous referees for their constructive comments. The author states that no funding source or sponsor has participated in the realization of this work.

\section{Competing interests}

The author declares that he has no competing interests.

\section{Authors' contributions}

The work as a whole is a contribution of the author. All authors read and approved the final manuscript.

\section{Publisher's Note}

Springer Nature remains neutral with regard to jurisdictional claims in published maps and institutional affiliations. Received: 21 November 2017 Accepted: 7 May 2018 Published online: 14 June 2018

\section{References}

1. Beesack, P.: Hardy's inequality and its extension. Pac. J. Math. 11, 39-61 (1961)

2. Hall, R.: Generalized Wirtinger inequalities, random matrix theory, and the zeros of the Riemann zeta-function. J. Number Theory 97, 397-409 (2002)

3. Lavoie, J., Osler, T., Tremblay, R.: Fractional derivatives and special functions. SIAM Rev. 18(2), 240-268 (1976)

4. Watson, G.: A Treatise on the Theory of Bessel Functions. Cambridge University Press, Cambridge (1922) 